\title{
вмј Global Health Inequity of healthcare access and use and catastrophic health spending in slum communities: a retrospective, cross-sectional survey in four countries
}

\author{
The Improving Health in Slums Collaborative
}

\begin{abstract}
To cite: The Improving Health in Slums Collaborative. Inequity of healthcare access and use and catastrophic health spending in slum communities: a retrospective, cross-sectional survey in four countries. BMJ Global Health 2021;6:e007265. doi:10.1136/ bmjgh-2021-007265
\end{abstract}

Handling editor Seye Abimbola

Received 24 August 2021 Accepted 23 October 2021

Check for updates

(c) Author(s) (or their employer(s)) 2021. Re-use permitted under CC BY. Published by BMJ.

\section{Correspondence to}

The Improving Health in Slums Collaborative;

s.i.watson@bham.ac.uk

\section{ABSTRACT}

Introduction Tracking the progress of universal health coverage (UHC) is typically at a country level. However, country-averages may mask significant small-scale variation in indicators of access and use, which would have important implications for policy choice to achieve UHC.

Methods We conducted a retrospective cross-sectional household and individual-level survey in seven slum sites across Nigeria, Kenya, Bangladesh and Pakistan. We estimated the adjusted association between household capacity to pay and report healthcare need, use and spending. Catastrophic health expenditure was estimated by five different methods. Results We surveyed 7002 households and 6856 adults. Gini coefficients were wide, ranging from 0.32 to 0.48 across the seven sites. The total spend of the top $10 \%$ of households was 4-47 times more per month than the bottom $10 \%$. Households with the highest budgets were: more likely to report needing care (highest vs lowest third of distribution of budgets: +1 to +31 percentage points (pp) across sites), to spend more on healthcare (2.0 to 6.4 times higher), have more inpatient and outpatient visits per year in five sites (1.0 to 3.0 times more frequently), spend more on drugs per visit (1.1 to 2.2 times higher) and were more likely to consult with a doctor (1.0 to 2.4 times higher odds). Better-off households were generally more likely to experience catastrophic health expenditure when calculated according to four methods ( -1 to $+12 \mathrm{pp})$, but much less likely using a normative method ( -60 to -80 pp).

Conclusions Slums have a very high degree of inequality of household budget that translates into inequities in the access to and use of healthcare. Evaluation of UHC and healthcare access interventions targeting these areas should consider distributional effects, although the standard measures may be unreliable.

\section{INTRODUCTION}

The aim of universal health coverage (UHC) is to ensure that everyone gets the care they need regardless of their ability to pay, and that no-one suffers undue financial hardship as a result of seeking healthcare. ${ }^{1}$ There has been a growth in the commitment worldwide to achieving UHC and it was adopted as one of the United Nations' Sustainable Development

\section{Key questions}

What is already known?

- We previously identified 20 narrative reviews and 128 cohort studies describing epidemiology determinants of health, and healthcare use in slum settings through a systematic search of MEDLINE and Embase in 2016, which showed that people living in slums encountered many barriers in accessing health services.

- While previous studies describe differences in provision of health services, for example, a preponderance of private providers in India and public facilities in Ethiopia and Kenya, they do not provide systematic detail or analysis of factors determining use of facilities including income, nor do they report distributional differences and inequalities in these neighbourhoods.

Goals (SDGs; indicator 3.8.2). Countries are responding by designing programmes to migrate towards UHC.

It is widely agreed that supply-side healthcare reforms should be pro-poor, including measures like elimination of user fees, ${ }^{2}$ introduction of public or social insurance schemes, ${ }^{3}$ or increased local public provision of services. ${ }^{4}$ However, identifying poor people specifically has limitations: it is costly, inaccurate and risks 'adverse selection'. As a result many programmes target 'people in poor neighbourhoods ${ }^{5}$ instead.

An archetypal 'poor neighbourhood' is a slum or informal settlement. Slums are often the target of localised healthcare interventions given the definitional lack of services and access to care in these neighbourhoods ${ }^{67}$; indeed, the UN's SDGs identifies addressing 'the plight of slums' as a global priority. UHC is a statement though about individual level rights and protections and so targeting and monitoring. However, targeting programmes at a slum-level only makes sense if the people living in slums are homogeneously poor relative to the country at large and if the response to intervention would be to improve healthcare access for all residents. 


\section{Key questions}

\section{What are the new findings?}

- We find evidence of significant inequalities in household expenditure across all the seven sites and that these inequalities in spending translate into inequalities in healthcare use with the best-off households visiting doctors and nurses up to three times as frequently as the worst-off.

- We also find evidence of high rates of catastrophic health expenditure, however, better-off households were more likely to experience catastrophic health expenditure according to four out of the five methods we used, suggesting that these measures were relatively poor at identifying 'affordability'.

\section{What do the new findings imply?}

- Broad neighbourhood-level policies to improve healthcare access may have the effect of exacerbating healthcare inequalities without specific support for the worst-off households.

- The distributional consequences of interventions should be routinely included in evaluations of healthcare access interventions to identify whether the worst-off households are benefitting.

There are several indicators widely used to monitor UHC progress, including examining the association between income and care use, adjusting for proxies for healthcare need, and examining catastrophic health expenditure (CHE) rates. CHE is most commonly measured as healthcare expenditures exceeding $10 \%$ or $25 \%$ of a household's total budget, measured either as income or consumption expenditure. ${ }^{8}$ At a country level, a low incidence of CHE may simply reflect low service coverage. ${ }^{4}$ However, at a more granular level (within country or community) better-off households may spend more on healthcare both in absolute and proportionate terms when faced with the same service coverage, so better-off households may also be more likely to be classified as experiencing CHE even though the expenditure was affordable for them. ${ }^{8}$ Thus, a slum may appear to be getting worse-off by some measures of CHE when in fact their material circumstances are improving, or better-off households use more healthcare, and service coverage remains unchanged with significant gaps for the poorest. Alternative methods of calculating CHE have been proposed to try to combat this effect, but the results can vary widely, and have seldom been examined at community levels. The only previous slum-specific study on CHE showed that the proportion of households experiencing CHE in two Kenyan slums varied between $2 \%$ and $28 \%$ depending on the method used ${ }^{10}$

Recent reports have reported generally positive progress internationally on UHC using these indicators at the country level. ${ }^{411} 12$ However, there exists little previous evidence on the values one might expect of indicators of inequitable access and financial hardship within a slum or similar community, nor whether the most commonly used measures can provide a useful insight into the performance of UHC programmes at this level. Thus, there remains questions about healthcare access in poor communities and how to monitor it.
In this study, we examine inequalities of healthcare need, access, use and expenditure within slums. We first consider income and household budget inequalities. Second, we estimate the association between these inequalities and reported healthcare need, access and use. And third, we estimate five indicators of CHE for each household and explore how they relate to each other and within-slum inequalities. Our objective is to provide both a reference for future evaluations of access-promoting interventions, and to consider the usefulness of such measures within these communities. We study seven slums across four countries with significant populations of urban poor residents: Nigeria, Kenya, Pakistan and Bangladesh.

\section{METHODS}

\section{Data collection}

We conducted a retrospective, cross-sectional household and individual survey across seven slum sites in Nigeria, Kenya, Bangladesh and Pakistan. We refer to these sites pseudonymously as NG1 and NG2 (Ibadan, Nigeria), NG3 (Lagos, Nigeria), KE1 and KE2 (Nairobi, Kenya), PK1 (Karachi, Pakistan) and BD1 (Dhaka, Bangladesh). Full details of the survey methodology are published elsewhere. $^{13}$

All sites were mapped using remotely-sensed data, which was then ground-truthed using a participatory mapping process, and any errors corrected. All resident households were listed in this stage to form a sampling frame, from which we drew a spatially-regulated ${ }^{14}$ random sample of 1200 households from each site, with the goal of achieving a sample of 1000 households. Field workers made up to three attempts to visit each sampled household. A member of each consenting household (typically the head of household) completed a survey on household-level information including a roster with demographic and socioeconomic information, and household income and spending across various categories (including rent, food, water, electricity and healthcare). An adult and a child under 12 were randomly sampled from the household roster to complete individual-level surveys. We sampled adult women at a ratio of 2:1 with respect to adult men as we hypothesised that women would use healthcare more frequently than men. The individual-level survey collected information on a range of healthcare need, use and spending. Data quality control procedures were used in the survey process including spot checks and sit-ins by field supervisors and computer checks of submitted data with any erroneous entries sent back to the field for correction. Surveys were translated using iterative process involving forward and independent backward translations.

\section{Patient and public involvement}

Mapping of the study sites, identification of healthcare facilities and enumeration of resident households was conducted using a participatory process involving local residents. Healthcare facility managers and owners were 
consulted about identification of their facilities. The public were not involved in the design of the survey questionnaires, however feedback was sought from residents in a pilot survey in all sites to assess the time burden of participating. Patient and public focus groups were established to present the findings, receive feedback and provide contextualising interpretation of the results.

\section{Statistical analysis}

Household budget

For every household we calculated its total budget, which we defined as the total monthly household consumption expenditure. We also calculated the household budget per equivalent person. 'Equivalent persons' were calculated using the square root of the number of household members to account for the economies of scale of living in larger households. ${ }^{15}{ }^{16}$ Households were divided into thirds within each site based on their position in the distribution of household budget per equivalent person (bottom, middle and top) for the adjusted analyses of association of household budget with healthcare need, use and spending.

\section{Budget inequalities}

To summarise household budget inequality within each site we calculated three measures: (i) the Gini coefficient for the total household budget; (ii) the ratio of the 90th to 10th percentiles of household budget (' $90 / 10$ ratio'); and (iii) the ratio of the 90th to 10th percentiles of household budget per equivalent person.

\section{Inequalities of need and use}

Individuals were asked if they had needed healthcare in the previous 12 months and if so whether they had received healthcare. We estimated absolute risk differences in the proportion of respondents reporting healthcare need and if they had received it by thirds of household budget per equivalent person adjusted for age, age squared, sex, highest level of education completed and whether the respondent had a long-term health condition.

At the household level we extracted healthcare spending in the previous month and estimated a log-linear model (log healthcare expenditure against household budget third) adjusted for number and age of household members. At the individual-level we also extracted: number of healthcare visits in the previous year not involving an overnight stay, number of visits with an overnight stay, whether a visit without an overnight stay was to a doctor and the amount spent on a visit without an overnight stay on consultancy fees, drugs and tests. For each of the individual-level outcomes we estimated a regression model separately by site (Poisson for count data for an incidence rate ratio, binomial-logistic for binary for an OR and log-linear for continuous for an elasticity) of each outcome on their household's budget third, adjusted for the individual's age, age squared, sex, household size, highest education level achieved and whether they had any long-term conditions.

\section{Catastrophic health expenditure}

Following Cylus $e t a l^{8}$ we calculated five measures of CHE for each study site. Each measure was defined based on the ratio of healthcare spending to the household's capacity to pay. In addition to the household's total budget described above, the other measures of household 'capacity to pay' were:

- Actual food spending: The total budget minus the household's actual monthly spending on food.

- Partially normative food: The total budget minus an amount representing subsistence food spending, except for households already below the subsistence amount for whom actual food spending was subtracted. Subsistence food spending was determined as the average food spending per equivalent person among households whose food share of total spending was between the 45 th and 55th percentile.

- Normative spending on food, rent and utilities. The total budget minus an amount representing subsistence spending on food, rent and utilities, which was defined as the mean spending on these items per equivalent person for households that were between the 25 and 35 percentiles of total budget.

The five CHE definitions were:

- Methods 1 and 2: $10 \%$ and 25\% of total budget, respectively (used by WHO's Global Health Observatory, World Bank, and others);

- Method 3: 40\% of actual food spending (used by Pan American Health Organisation and World Bank);

- Method 4: $40 \%$ of partially normative food spending (used by WHO)

- Method 5: 40\% of normative spending on food, rent and utilities (used by WHO's Regional Office for Europe).

We examined the proportion of households identified as experiencing CHE for each method and we determined their agreement as the proportion of households classified in the same way by each method. Finally, we estimated absolute risk differences in the proportion of households with CHE by thirds of household budget per equivalent person adjusted for the number and age of household members.

\section{RESULTS}

Table 1 reports summary statistics of the study populations estimated from the samples. Overall, we interviewed 7002 households and 6857 adults. The seven sites were broadly comparable in demographic characteristics, although the Nigerian sites had moderately higher educational levels than elsewhere, and employment rates were lower in sites KE1 and PK1 (55\% and 46\%, respectively) than elsewhere $(61 \%-70 \%)$. 


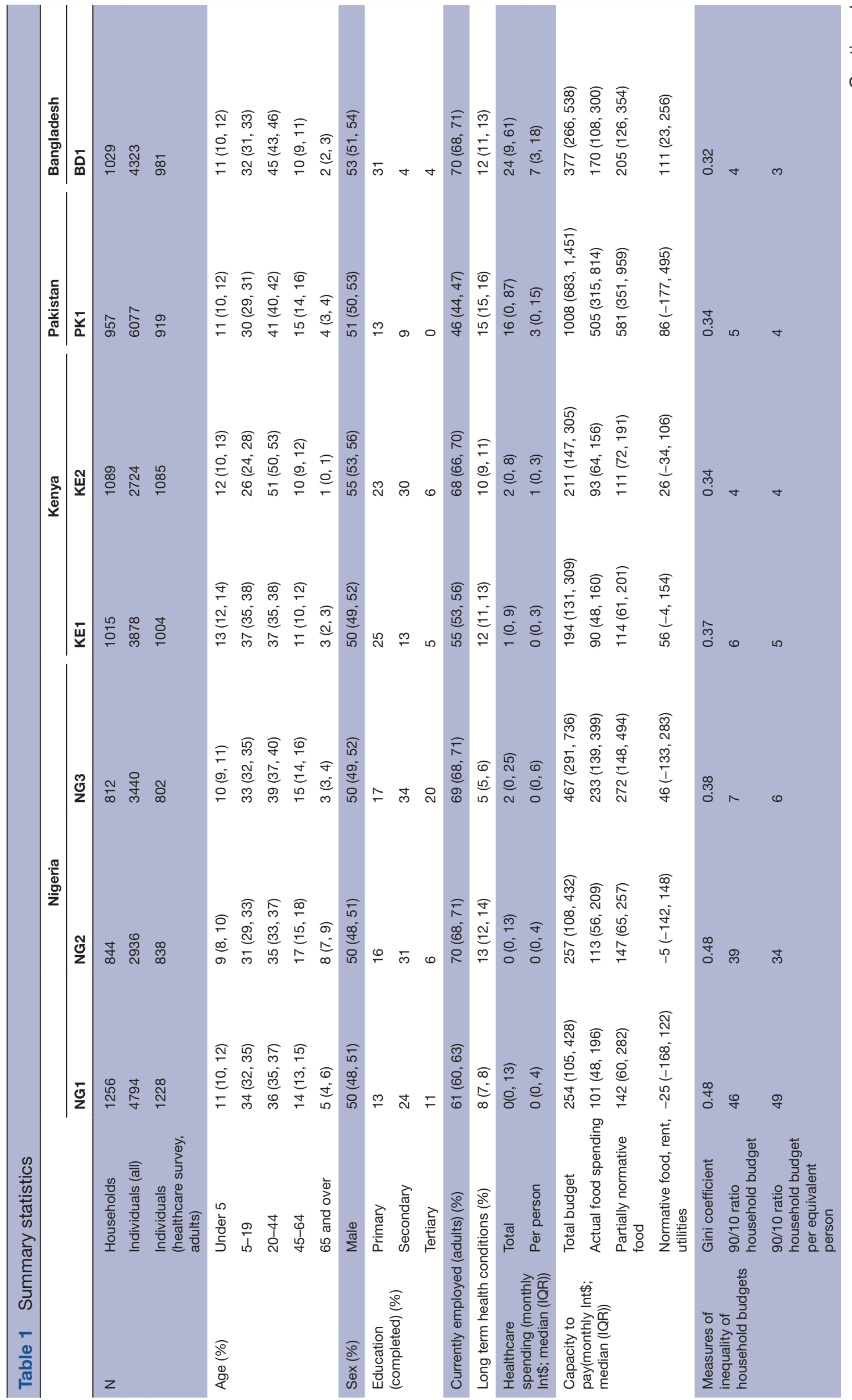

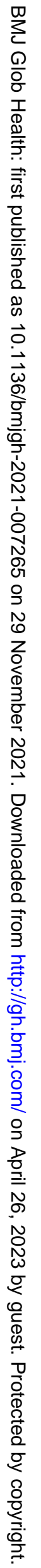




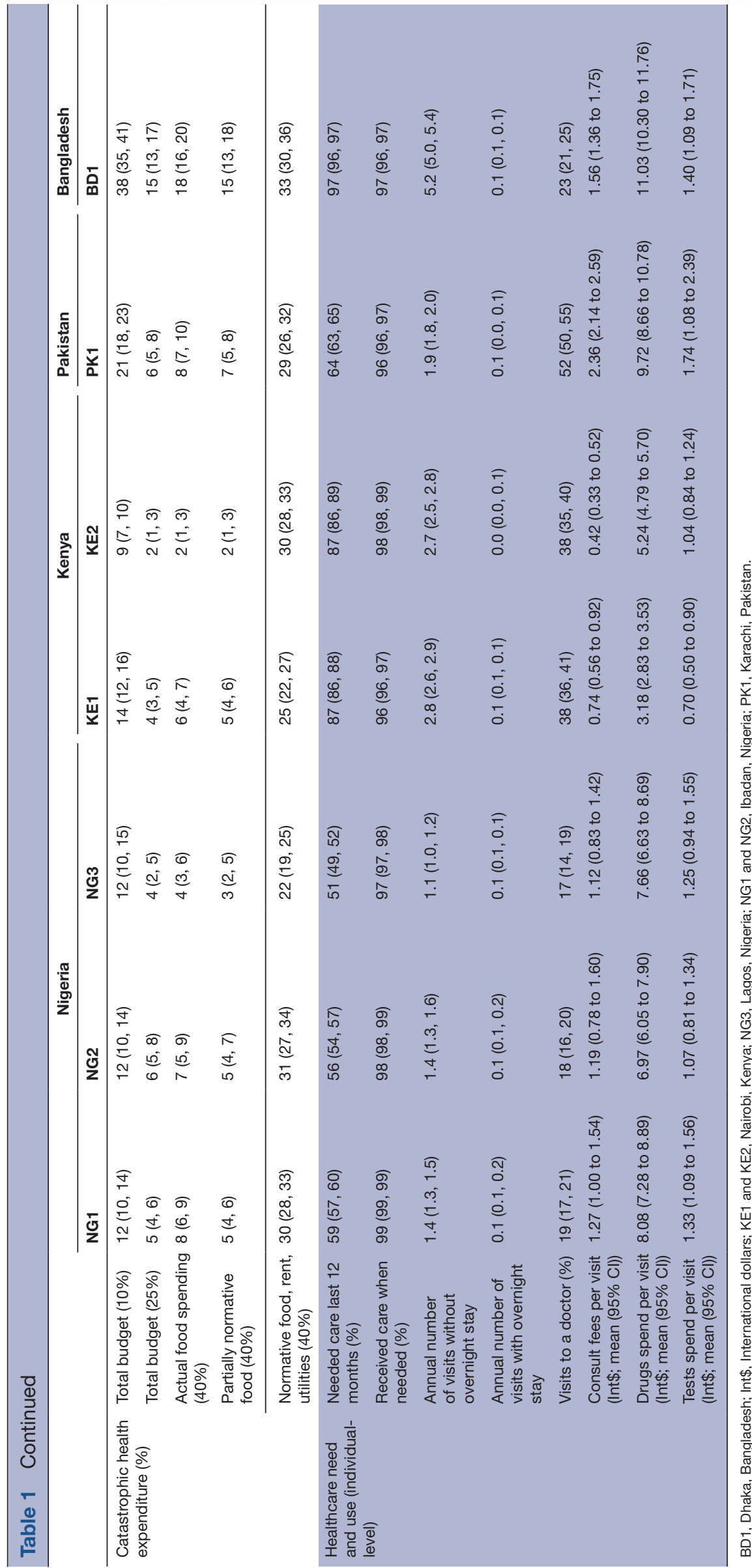




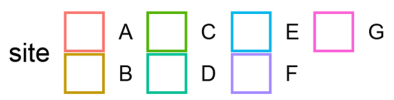

\section{A Capacity to pay}

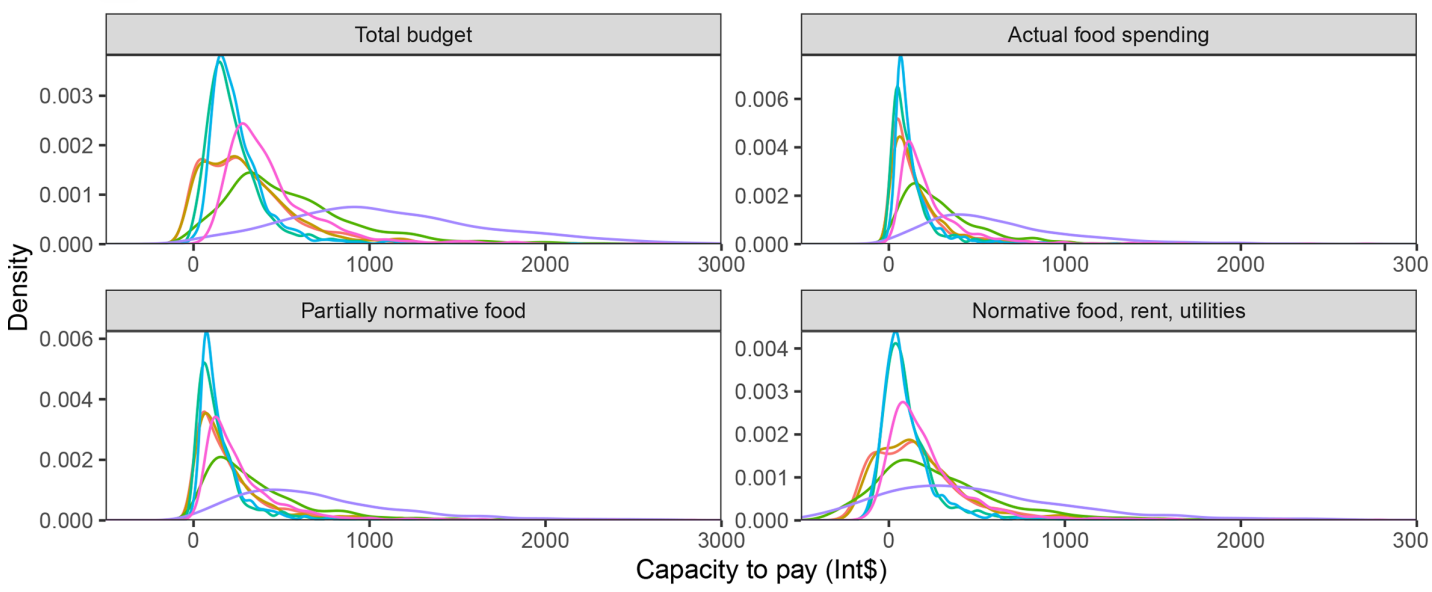

B Capacity to pay per equivalent person

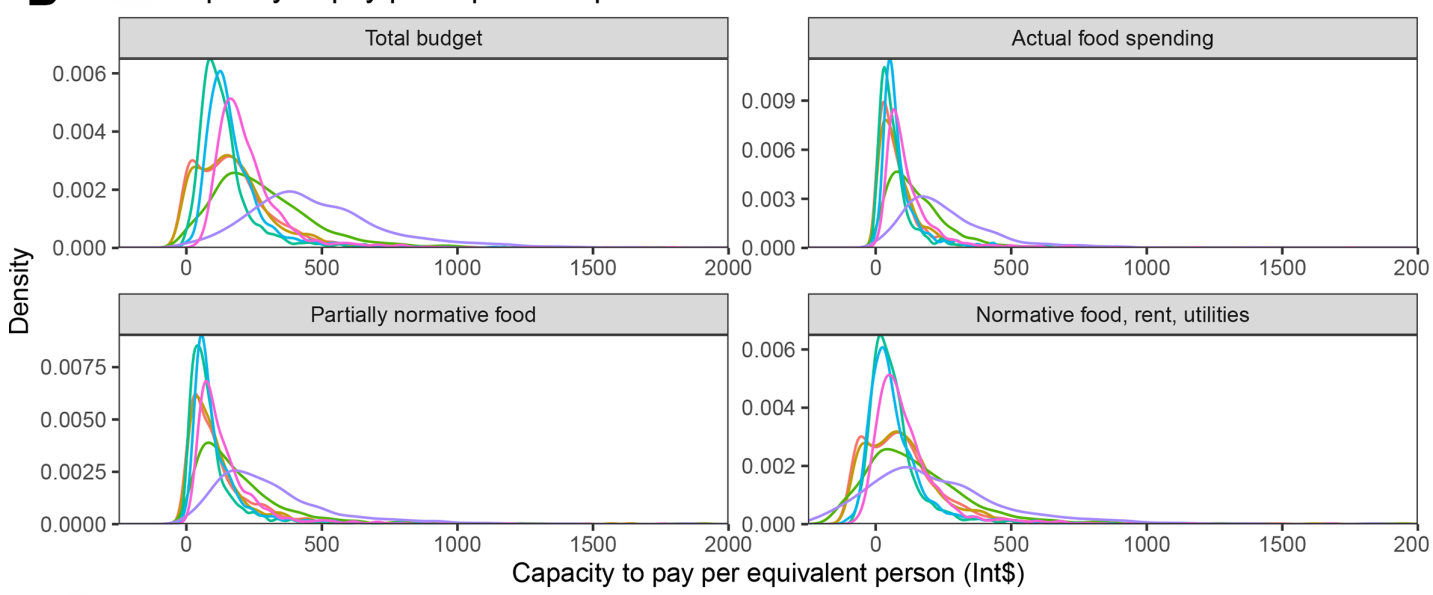

C Proportion of capacity to pay spent on healthcare

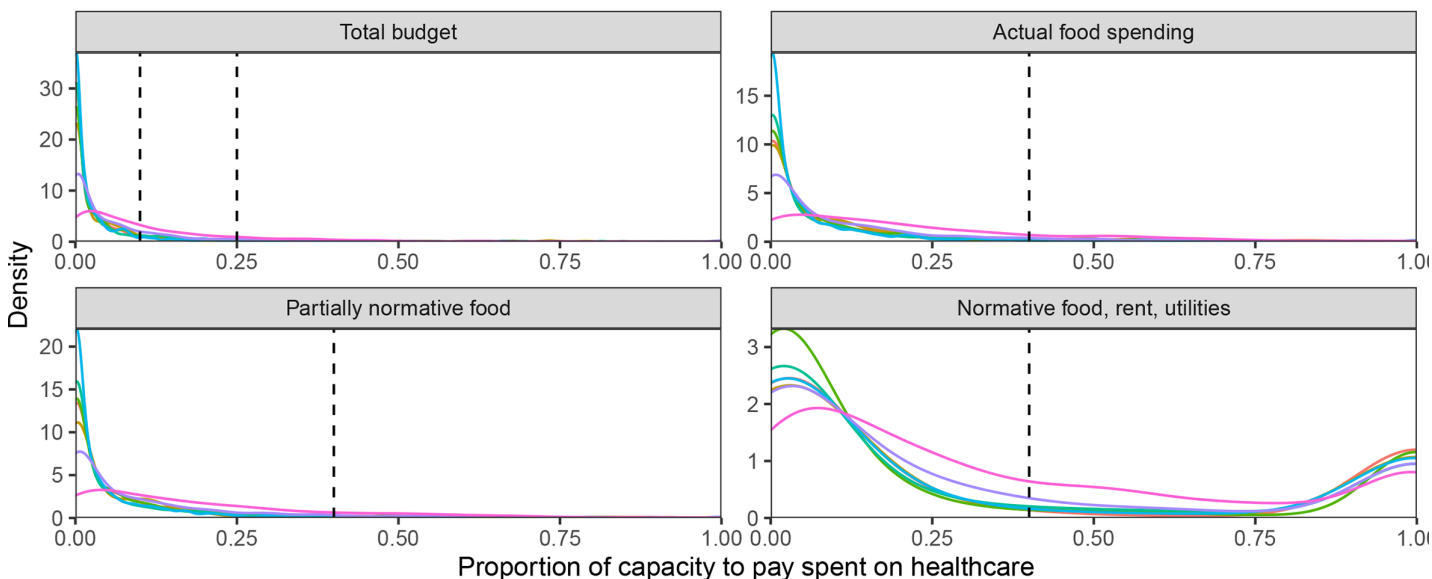

Figure 1 (A) Density plot of the different measures of capacity to pay for each site. (B) Density plot of the different measures of capacity to pay divided by the number of equivalent persons in each household. (C) Density plot of the proportion of capacity to pay spent on healthcare with vertical lines indicating catastrophic health expenditure thresholds. Int\$, International dollars

\section{Household budget inequality}

Figure 1A,B show the different measures of capacity to pay. Median household total budget was highest in Pakistan (International dollars (Int\$) 1008 vs Int\$194-467 elsewhere). While households had approximately double the number of members in PK1 compared with other sites, it still had the highest median total budget per equivalent person. Gini coefficients were wide ranging 
Table 2 Adjusted absolute risk differences (percentage point) in the probability of reporting a need for healthcare in the previous 12 months by tertile of household consumption expenditure per equivalent person

\begin{tabular}{llllllll}
\hline Adults & NG1 & NG2 & NG3 & KE1 & KE2 & PK1 & BD1 \\
\hline Bottom & Ref. & Ref. & Ref. & Ref. & Ref. & Ref. & Ref. \\
Middle & 22.6 & 7.0 & 7.3 & 10.6 & -1.8 & 7.0 & -0.1 \\
& $(16.3,28.9)$ & $(-0.5,14.4)$ & $(-0.4,15.0)$ & $(4.4,16.8)$ & $(-7.0,3.5)$ & $(-1.3,14.1)$ & $(-2.5,2.3)$ \\
Top & 31.0 & 14.1 & 7.3 & 11.0 & 2.2 & 4.7 & 0.6 \\
& $(16.3,28.9)$ & $(6.6,21.6)$ & $(-0.5,15.0)$ & $(4.8,17.3)$ & $(-3.2,7.5)$ & $(-2.4,11.9)$ & $(-1.9,3.0)$ \\
Children & NG1 & NG2 & NG3 & KE1 & KE2 & PK1 & BD1 \\
Bottom & Ref. & Ref. & Ref. & Ref. & Ref. & Ref. & Ref. \\
Middle & -3.7 & 5.6 & -15.4 & 0.9 & 7.1 & 5.7 & 4.2 \\
& $(-19.5,12.3)$ & $(-17.0,28.1)$ & $(-35.7,5.0)$ & $(-5.0,6.9)$ & $(-0.2,14.4)$ & $(-2.8,14.2)$ & $(0.8,7.5)$ \\
Top & -16.0 & -4.0 & -1.7 & 4.6 & 4.1 & 4.9 & 1.5 \\
& $(-32.2,0.3)$ & $(-26.8,18.8)$ & $(-24.9,21.4)$ & $(-1.9,11.0)$ & $(-4.5,12.7)$ & $(-3.7,13.6)$ & $(-1.9,4.9)$ \\
\hline
\end{tabular}

BD1, Dhaka, Bangladesh; KE1 and KE2, Nairobi, Kenya; NG3, Lagos, Nigeria; NG1 and NG2, Ibadan, Nigeria; PK1, Karachi, Pakistan.

with respect to total household budgets: from 0.32 in BD1 to 0.48 in NG1 and NG2. The 90/10 ratios for sites NG1 and NG2 were 46 and 39, indicating the top $10 \%$ of households spent 46 and 39 times more per month than the bottom $10 \%$, respectively, in these sites. These values were much greater than the other sites, which nevertheless ranged from 4 to 7 .

\section{Inequalities of need and use of health services}

Healthcare need

In Kenya and Bangladesh, the vast majority of respondents reported requiring healthcare at any point in the previous year (87\% in Kenya and 97\% in Bangladesh) whereas in Nigeria and Pakistan the proportions were lower (51\%-64\%) (table 1). However, in all countries $96 \%$ or more reported receiving care when they perceived a need for it. Table 2 reports adjusted risk differences for the probability of reporting needing care by third of the distribution of total budget per equivalent person. Households with the highest budgets were more likely to report needing care (highest vs lowest third: 0.6 to 31.0 percentage points higher), except for in Bangladesh where, as stated, almost all reported needing care. The differences were greatest in sites NG1 and NG2. There was little evidence that children were more likely to report needing care in the best-off households (eg, highest to lowest thirds: -16.0 to 4.9 percentage points). We did not estimate models for receiving care if needed as there was little to no variation in the outcome, given that $98 \%$ or more reported receiving care when a health need was perceived.

Overall health spending

Better-off households spent more both absolutely and proportionately on healthcare in all sites after adjusting for socio-demographic characteristics. Figure 2 shows the estimated proportionate difference in healthcare spending between thirds of the distribution of household

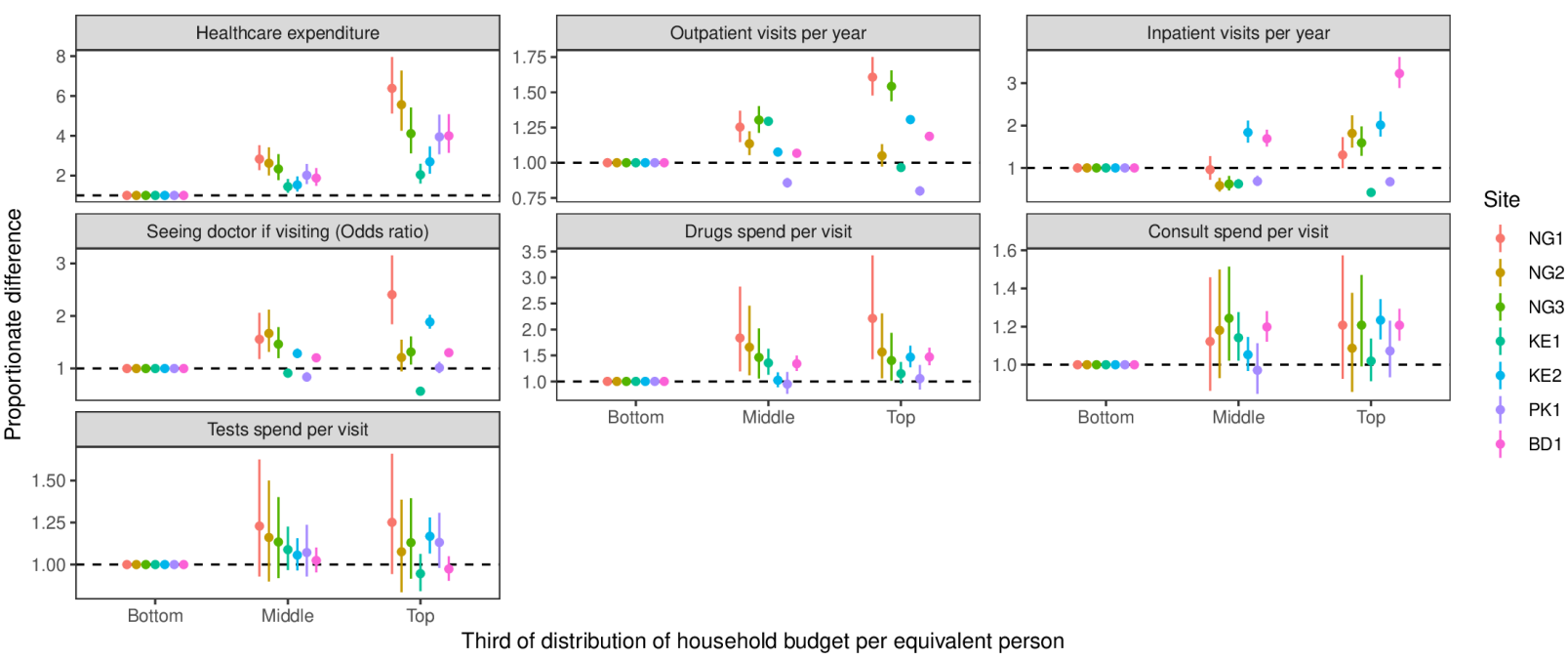

Figure 2 Adjusted proportionate difference in healthcare use and expenditure, overall and per visit, between thirds of the distribution of household budget per equivalent person ('Bottom' is reference category). BD1, Dhaka, Bangladesh; KE1 and KE2, Nairobi, Kenya; NG1 and NG2, Ibadan, Nigeria; NG3, Lagos, Nigeria; PK1, Karachi, Pakistan. 
budget per equivalent person adjusted for age, sex and other characteristics. The top third of households spent 2.0 to 6.4 times more than those in the bottom third.

Type of healthcare use

Adjusted inpatient and outpatient visit rates were higher in Nigeria, Pakistan and Bangladesh for better-off households (top vs bottom third: 1.0 to 3.0 times more frequently), and patients in these locations were more likely to see a doctor when they sought care (1.0 to 2.4 times higher odds). The mean spending on drugs per visit was higher for better-off households in all sites (1.1 to 2.2 times higher), however there was little evidence of a difference in spending on consultation fees and tests per visit.

\section{Catastrophic health expenditure Comparison of methods}

Table 1 and figure 1A,B report the capacities to pay for healthcare within each site. Site PK1 had the highest capacity to pay according to all measures, except for normative spending on food, rent and utilities (method 5 ), in which BD1 had the highest capacity to pay. Sites NG1 and NG2 had a negative median capacity to pay using the normative method (method 5) (Int \$-25 and Int \$-5). The African sites were all lower than PK1 and BD1 for all measures.

Figure 1C shows the distribution of the proportion of each capacity to pay spent on healthcare within each site and table 1 reports the proportions of households classified as experiencing CHE using each of the five methods. For methods based on household budget, actual food spending and partially normative spending (methods 1-4), between $2 \%$ and $18 \%$ of households had CHE. Bangladesh was also the highest on each of these measures (15\%-18\% versus $2 \%-8 \%$ elsewhere). However, for the normative spending method (method 5 ), between $24 \%$ and 33\% of all households had CHE and Bangladesh had comparable rates to the other sites. Table 1 also shows the reported mean spending per outpatient visit (including clinic, hospitals and pharmacies); the costs in Bangladesh were comparable to other sites, however the visit rate was about twice as frequent as elsewhere (5.2 vs 1.1 to 2.8 visits per person-year elsewhere). Table 3 shows the agreement between the different methods. Methods 1-4 classify between $88 \%$ and $98 \%$ of the households in the same way, however the agreement between method 5 and methods $1-4$ was $78 \%$ to $82 \%$ so that approximately $20 \%$ of households were classified differently by method 5 .

\section{Catastrophic health expenditure by household budget}

Figure 3 shows the relationship between household budget and CHE. There was evidence that, for all but the normative method (method 5), better-off households were more likely to experience CHE particularly using method 1, although the differences were variable $(-1$ percentage points $(\mathrm{pp})$ to $12 \mathrm{pp}$ higher in top third vs bottom third) and not consistent between sites. According to the normative method, households in the top third were $60-80 \mathrm{pp}$ less likely to experience CHE than those in the bottom third, suggesting the normative method identified worse-off households in particular.

\section{DISCUSSION}

\section{Economic variation in slums}

These slums are relatively small communities with populations ranging between 5000 and 60000 people. Yet there exists a wide distribution of household budgets within slum sites. The Gini coefficients for the sites ranged from 0.33 to 0.48 and the $90 / 10$ ratio was $4-7$ in five sites and over 30 in two Nigerian sites. For comparison, among the Organisation of Economic Cooperation and Development (OECD) countries, Gini coefficients for individual metropolitan areas have been estimated to range from approximately 0.20 to $0.45,{ }^{17}$ and in the UK the $90 / 10$ ratio of incomes was approximately $4 .{ }^{18}$ While comparable figures are not available for low and middle income country (LMIC) metropolitan areas, the results suggest that slums are not concentrated areas of extreme poverty within a city, but have a heterogeneous population with inequality comparable to that observed at even the level of whole countries.

\section{Financial circumstances and healthcare need and use}

The inequality in household financial circumstances translates into inequities in healthcare access and use

Table 3 Percentage agreement between different methods of identifying catastrophic health expenditure

\begin{tabular}{|c|c|c|c|c|c|}
\hline & Total budget (10\%) & $\begin{array}{l}\text { Total budget } \\
(25 \%)\end{array}$ & $\begin{array}{l}\text { Actual food } \\
\text { spending }(40 \%)\end{array}$ & $\begin{array}{l}\text { Partially normative } \\
\text { food }(40 \%)\end{array}$ & $\begin{array}{l}\text { Normative } \\
\text { food, rent, } \\
\text { utilities } \\
(40 \%)\end{array}$ \\
\hline Total budget (10\%) & - & & & & \\
\hline Total budget (25\%) & $88 \%$ & - & & & \\
\hline Actual food spending (40\%) & $91 \%$ & $97 \%$ & - & & \\
\hline $\begin{array}{l}\text { Partially normative food } \\
(40 \%)\end{array}$ & $89 \%$ & $98 \%$ & $98 \%$ & - & \\
\hline $\begin{array}{l}\text { Normative food, rent, utilities } \\
(40 \%)\end{array}$ & $78 \%$ & $78 \%$ & $80 \%$ & $82 \%$ & - \\
\hline
\end{tabular}




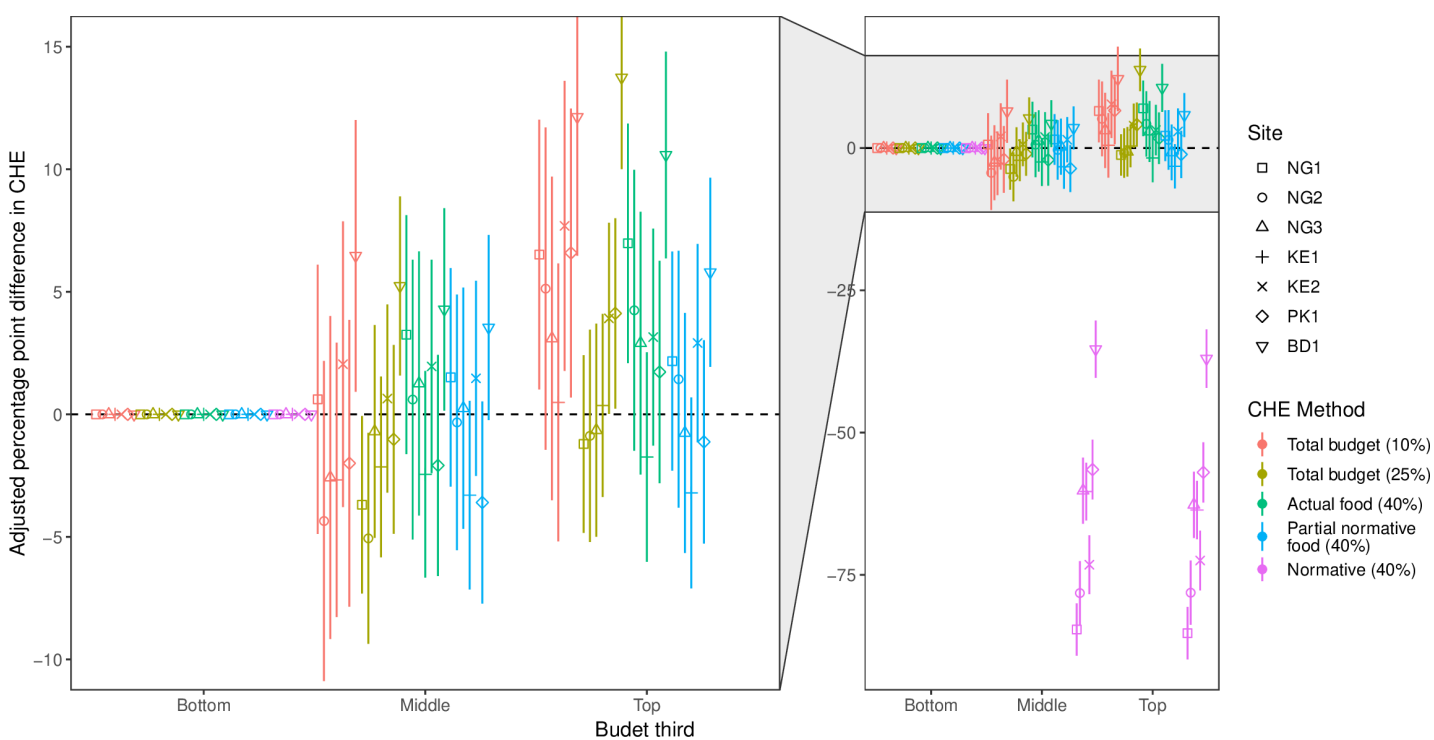

Figure 3 Adjusted percentage point difference in the proportion of the population experiencing catastrophic health expenditure between thirds of the distribution of household budget per equivalent person. The left-hand panel is a zoom in of the main plot on the right where indicated, with the results of the first four methods. BD1, Dhaka, Bangladesh; CHE, catastrophic health expenditure; KE1 and KE2, Nairobi, Kenya; NG1 and NG2, Ibadan, Nigeria; NG3, Lagos, Nigeria; PK1, Karachi, Pakistan.

as worse-off households seek healthcare less frequently. Our results suggest that differences in use between individuals from better and worse-off households are explained by individuals as differences in their perceived need for healthcare rather than differences in their ability to access it or actual need for healthcare, particularly since in all our sites there were a wide range of public and private providers available within short distances. ${ }^{19}$ The differential recognition and assessment of health needs by income and education has been well documented both between and within countries $^{2021}$; here we show this likely extends even within small communities. Several mechanisms may be at play, including different expectations about health status, the role of education in the recognition of symptoms or the willingness to recognise symptoms when there are competing work needs.

Low healthcare use provides fewer opportunities to identify signs of non-communicable illness, such as cancers, which present in stage 3 or 4 despite the availability of diagnostic and therapeutic technology that could have identified and treated the cancer in earlier stages. ${ }^{22}{ }^{23}$ We would hypothesise therefore that the inequalities in budget, and hence healthcare use, translate into inequalities in the prognosis of diseases like cancers even within small communities like slums.

\section{Should we target UHC initiatives at the slum-level?}

In addition to differences in the recognition and reporting of symptoms, the propensity to seek care may also be lower among worse off households due to the relative costs of doing so. Across the African continent, in a large number of countries, survey evidence shows that households with greater wealth use healthcare services more frequently, but not because they are more likely than poorer people to benefit from accessing those services. ${ }^{24}{ }^{25}$ Similar evidence has been shown for South Asian and Southeast Asian countries. Our results are focused at a much smaller scale, but they tell the same story.

The inequality of income and healthcare use in slums suggests that the 'slum' categorisation may be of limited value in directing UHC policy. Some authors have suggested that improved education among the poor may be a solution to these inequalities in use of health services to better enable people to perceive healthcare needs. ${ }^{24} 26$ However, even in our sites with reasonably homogeneous education levels, the same patterns were observed, and even after adjusting for education levels, individuals from better-off households were more likely to report experiencing a need for healthcare and consulting.

\section{Should we use CHE to monitor local UHC progress?}

CHE is a widely used UHC indicator of health systems functioning designed to identify the incidence of financial hardship arising due to healthcare use. It was used in a recent global survey of 'progress towards UHC'. However, our results echo those of Cylus et $a l^{8}{ }^{8}$ who showed that the most widely used methods of calculating CHE reflect lower barriers to use healthcare for better-off households rather than hardship or affordability.

Recent work has shown a global increase in the proportion of households spending over $10 \%$ and $25 \%$ of their budgets on healthcare, ${ }^{11}$ although it is not clear whether this is because of growth in global incomes or worsening financial protections and public support for healthcare (or both). One alternative measure we examined was a fully normative method that subtracted a context-specific 
amount needed for subsistence. The subsistence amount is based on an arbitrary cut-off in the distribution of spending in the community, but it discriminated between better-off and worse-off households with many households having zero or negative capacity to pay. However, as with all the methods, it is not clear if households identified as having CHE faced unaffordable spending. Households evidently made choices about their need for and use of healthcare based on their financial circumstances.

Households who face the sudden and devastating effects of diseases like tuberculosis or trauma will likely face unaffordable healthcare costs. CHE may be a useful measure of the distress caused by these particular circumstances. But as an aggregate measure of access to primary care, CHE is highly non-specific, variable and dependent on the methodology used, suggesting it is a poor measure for tracking UHC at local levels. CHE methods and their results are also affected by length of recall period of the questions and the level of disaggregation of spending in surveys, ${ }^{27} 28$ further raising questions about their validity.

\section{Strengths and limitations}

We acknowledge several limitations to the result presented here. We did not assess the actual health status of the participants, so we cannot identify whether the differences in reported need and use reflect actual need. Given previous findings that indicate individuals who have a lower level of education or income are less likely to recognise illness and to report lower levels of severity, ${ }^{20}$ we have assumed that our results indicate inequitable differences in healthcare use rather than differences in health status. However, further research should address this topic. While every effort was made to ensure a representative sample, our response rates ranges from $70 \%$ to $95 \%$ across the study sites. Individuals in poorer health or from worse-off households may have been less likely to respond ${ }^{29}{ }^{30}$, which may bias our results. However, we suggest that this may lead us to underestimate the degree of inequality in these communities.

\section{CONCLUSIONS}

Our results suggest that the evaluation of any locally targeted policy to improve healthcare access and use, such as subsidies, clinical provision or otherwise, should consider the distribution and heterogeneity of effects across the targeted population and not just the mean effect. Some policies or interventions to improve access could result in widening inequalities and leave the very poorest behind. Strategies to improve use of health services will likely have to focus at very granular levels to be optimally effective since large-scale solutions, such as public funding of all healthcare free at the point of use, are likely infeasible for many LMICs presently. While some reports recognise the heterogeneity in slum populations, 'slums' are often treated in the academical and policy literature as an archetypal grouping of poor people. ${ }^{5}$ However, they are complex, heterogeneous areas with highly variable characteristics ${ }^{31}$ and it is unlikely that broad 'slum-level' policy solutions would be as successful as those that take a more granular view of the intended beneficiaries of these policies.

Collaborators The Improving Health in Slums Collaborative: African Population and Health Research Centre (APHRC), Nairobi, Kenya-Pauline Bakibinga, Caroline Kabaria, Ziraba Kasiira, Peter Kibe, Catherine Kyobutungi, Nelson Mbaya, Blessing Mberu, Shukri Mohammed and Anne Njeri; Aga Khan University, Karachi, Pakistan—lqbal Azam, Romaina Iqbal, Ahsana Nazish and Narijis Rizvi; Independent University, Bangladesh, Dhaka, Bangladesh—Syed A K Shifat Ahmed, Nazratun Choudhury, Ornob Alam, Afreen Zaman Khan, Omar Rahman and Rita Yusuf; Nigerian Academy of Sciences, Lagos, Nigeria-Doyin Odubanjo; University of Ibadan, Ibadan, Nigeria-Motunrayo Ayobola, Olufunke Fayehun, Akinyinka Omigbodun, Mary Osuh, Eme Owoaje and Olalekan Taiwo; University of Birmingham, Birmingham, UK—Richard J Lilford (PI), Jo Sartori and Samuel I Watson; University of Lancaster, Lancaster, UK—Peter J Diggle; University of Warwick, Coventry, UK — Navneet Aujla, Yen-Fu Chen, Paramjit Gill, Frances Griffiths, Bronwyn Harris, Jason Madan, Helen Muir, Oyinlola Oyebode, Vangelis Pitidis, João Porto de Albuquerque, Simon Smith, Celia Taylor, Philip Ulbrich, Olalekan A Uthman, Ria Wilson and Godwin Yeboah.

Contributors The corresponding author and principal investigator drafted the manuscript and conducted the analyses. All members of the collaborative were involved in the design of the project, data collection, data analysis and approved the final manuscript. SW is the guarantor.

Funding The project was funded by the National Institutes for Health Research (16/136/87).

\section{Competing interests None declared.}

Patient and public involvement Patients and/or the public were involved in the design, or conduct, or reporting, or dissemination plans of this research. Refer to the Methods section for further details.

\section{Patient consent for publication Not applicable.}

Ethics approval The NIHR Global Health Research Unit on Improving Health in Slums was granted full ethical approval by the University of Warwick Biomedical and Scientific Research Ethics Sub-Committee (REG0-2017-2043 AM01), the Ministry of Health, Lagos State Government (LSMH/2695/11/259) the Ministry of Health, Oyo State Government (ADB/479/657), Amref Health Africa (AMREF-ESRC P440/2018), the National Bioethics Committee Pakistan (4-87/NBC-298/18/RDC3530) and the Bangladesh Medical Research Council. All the participants included in the study gave informed consent. All experimental procedures were performed in accordance with the guidelines and regulations followed relevant guidance as detailed in the manuscript.

Provenance and peer review Not commissioned; externally peer reviewed.

Data availability statement Data are available upon reasonable request. The data are available from the study authors on request.

Open access This is an open access article distributed in accordance with the Creative Commons Attribution 4.0 Unported (CC BY 4.0) license, which permits others to copy, redistribute, remix, transform and build upon this work for any purpose, provided the original work is properly cited, a link to the licence is given, and indication of whether changes were made. See: https://creativecommons.org/ licenses/by/4.0/.

\section{REFERENCES}

1 Hogan DR, Stevens GA, Hosseinpoor AR, et al. Monitoring universal health coverage within the sustainable development goals: development and baseline data for an index of essential health services. Lancet Glob Health 2018;6:e152-68.

2 Yates $\mathrm{R}$. Universal health care and the removal of user fees. Lancet 2009;373:2078-81

3 Xu K, Evans DB, Carrin G, et al. Protecting households from catastrophic health spending. Health Aff 2007;26:972-83.

4 Wagstaff A, Neelsen S. A comprehensive assessment of universal health coverage in 111 countries: a retrospective observational study. Lancet Glob Health 2020;8:e39-49.

5 Brelsford C, Lobo J, Hand J, et al. Heterogeneity and scale of sustainable development in cities. Proc Natl Acad Sci U S A 2017;114:8963-8. 
6 Lilford RJ, Oyebode O, Satterthwaite D, et al. Improving the health and welfare of people who live in slums. Lancet 2017;389:559-70.

7 Ezeh A, Oyebode O, Satterthwaite D, et al. The history, geography, and sociology of slums and the health problems of people who live in slums. Lancet 2017;389:547-58.

8 Cylus J, Thomson S, Evetovits T. Catastrophic health spending in Europe: equity and policy implications of different calculation methods. Bull World Health Organ 2018;96:599-609.

9 World Health Organization. Global health Observatory, 2021. Available: https://www.who.int/data/gho [Accessed 6 Jun 2021].

10 Buigut S, Ettarh R, Amendah DD. Catastrophic health expenditure and its determinants in Kenya slum communities. Int J Equity Health 2015;14:46.

11 Wagstaff A, Flores G, Hsu J, et al. Progress on catastrophic health spending in 133 countries: a retrospective observational study. Lancet Glob Health 2018;6:e169-79.

12 Wagstaff A, Flores G, Smitz M-F, et al. Progress on impoverishing health spending in 122 countries: a retrospective observational study. Lancet Glob Health 2018;6:e180-92.

13 Improving Health in Slums Collaborative. A protocol for a multi-site, spatially-referenced household survey in slum settings: methods for access, sampling frame construction, sampling, and field data collection. BMC Med Res Methodol 2019;19:109.

14 Chipeta M, Terlouw D, Phiri K, et al. Inhibitory geostatistical designs for spatial prediction taking account of uncertain covariance structure. Environmetrics 2017;28:e2425.

15 Poverty RR. Household size and child welfare in India. Econ Polit Wkly 2000;35:3511-20.

16 Meenakshi JV, Ray R. Impact of household size and family composition on poverty in rural India. J Policy Model 2002;24:539-59.

17 Boulant J, Brezzi M, Veneri P. Income levels and inequality in metropolitan areas: a comparative approach in OECD countries, 2016.

18 Office for National Statistics. Household income inequality, UK Fiancial year ending 2020, 2020. Available: https://www.ons.gov.uk/ peoplepopulationandcommunity/personalandhouseholdfinances/ incomeandwealth/bulletins/householdincomeinequalityfinancial/fina ncialyearending2020 [Accessed 30 Jun 2021].

19 Improving Health in Slums Collaborative, Watson S. Pharmacies in informal settlements: a retrospective, cross-sectional household and health facility survey in four countries. BMC Health Serv Res 2021;21:945
20 d'Uva TB, O'Donnell O, van Doorslaer E. Differential health reporting by education level and its impact on the measurement of health inequalities among older Europeans. Int $J$ Epidemiol 2008;37:1375-83.

21 Adesanya A O, Rojas BM, Darboe A, et al. Socioeconomic differential in self-assessment of health and happiness in 5 African countries: finding from world value survey. PLoS One 2017;12:e0188281

22 Rivera-Franco MM, Leon-Rodriguez E. Delays in breast cancer detection and treatment in developing countries. Breast Cancer 2018;12:117822341775267.

23 Stewart TS, Moodley J, Walter FM. Population risk factors for latestage presentation of cervical cancer in sub-Saharan Africa. Cancer Epidemiol 2018;53:81-92.

24 Bonfrer I, van de Poel E, Grimm M, et al. Does the distribution of healthcare utilization match needs in Africa? Health Policy Plan 2014;29:921-37.

25 Gordon T, Booysen F, Mbonigaba J. Socio-economic inequalities in the multiple dimensions of access to healthcare: the case of South Africa. BMC Public Health 2020;20.

26 Mulyanto J, Kringos DS, Kunst AE. Socioeconomic inequalities in healthcare utilisation in Indonesia: a comprehensive survey-based overview. BMJ Open 2019;9:e026164.

27 Lu C, Chin B, Li G, et al. Limitations of methods for measuring outof-pocket and catastrophic private health expenditures. Bull World Health Organ 2009;87:238-44.

28 Raban MZ, Dandona R, Dandona L. Variations in catastrophic health expenditure estimates from household surveys in India. Bull World Health Organ 2013;91:726-35.

29 Lallukka T, Pietiläinen O, Jäppinen S, et al. Factors associated with health survey response among young employees: a register-based study using online, Mailed and telephone interview data collection methods. BMC Public Health 2020;20:184.

30 Ekholm O, Gundgaard J, Rasmussen NKR, et al. The effect of health, socio-economic position, and mode of data collection on non-response in health interview surveys. Scand J Public Health 2010;38:699-706.

31 Lilford R, Kyobutungi C, Ndugwa R, et al. Because space matters: conceptual framework to help distinguish slum from non-slum urban areas. BMJ Glob Health 2019;4:e001267. 